\title{
Modeling and Performance Evaluation of Different CDMA Schemes for Terrestrial and Satellite Communication Systems
}

\author{
Enrico Del Re, Romano Fantacci, and Antonio Pazzaglia \\ Dipartimento di Ingegneria Elettronica \\ Università di Firenze \\ Via S. Marta, 3, 50139 Firenze, ITALY
}

\begin{abstract}
This paper deals with the modeling and performance evaluation of different Code Division Multiple Access schemes suitable for applications in terrestrial and satellite mobile communication networks. The paper is focused on the use of particular propagation channel models and the relative sensitivity of different demodulation schemes to their parameters. The main goal is to analyze how the increased interference level, typical of a multipath environment, can influence different demodulator architectures in different propagation conditions. Some issues concerning the characterization of the propagation channel models in the case of the RAKE receiver are also investigated and discussed.
\end{abstract}

\section{INTRODUCTION}

It can be shown that, taking advantage of the interference reduction induced by some techniques such as multibeamed or multisectored antennas and voice activation, CDMA is able to increase capacity with respect to FDMA and TDMA [1] However, these considerations contrast sharply with those of Turin who found that the environmental characteristics where the mobile link is established have a "multiplying" effect on multiple access interference, thereby sometimes resulting in an unacceptable quality degradation of the received signal [2].

Therefore, in order to evaluate true effectiveness, computeraided modeling is the only practical approach to carry out in deriving the performance of CDMA communication systems in a simple and a quick way.

This paper is concerned on the performance evaluation of different CDMA demodulator schemes in satellite and terrestrial fading channels on the basis of simulation results.

The organization of the paper is the following: after this introduction (Section I), Section II provides an expression for the transmitted signal. Section III illustrates the channel models while Section IV describes the coherent and differential receivers. Section $\mathrm{V}$ provides the performance analysis. Section VI evaluates the effectiveness of a simple RAKE demodulation algorithm and deals with the problems concerning the computer simulation of such a system. Section VII presents the conclusions.

\section{TRANSMITTER MODEL}

Let us consider the situation of $M$ active transmitters at the base station.

Under these assumptions, the complex envelope of the signal transmitted by the base station (satellite or terrestrial) can be defined as [3]

$$
S(t)=\sum_{k=1}^{M} \sum_{i=-\infty}^{\infty}\left(c_{p, i \mid t_{N}}^{k} d_{p,(i)_{N}}^{k}+j c_{q,|i|_{N}}^{k} d_{q,(i)_{N}}^{k}\right) g_{T}\left(t-i T_{c}\right)
$$

\section{where:}

$$
\begin{aligned}
& |\cdot|_{N}=\cdot \operatorname{modulus} N \\
& \{\cdot\}_{N} \propto \operatorname{int}\left\{\frac{\cdot}{N}\right\}
\end{aligned}
$$

$d_{p, n}^{\star} \quad$ sign of the $k t h$ user $n t h$ in-phase transmitted symbol $d_{a, x}^{*} \quad$ sign of the $k t h$ user $\boldsymbol{n} t h$ quadrature transmitted symbol $c_{p, k}^{q, \pi} \quad$ sign of the $h t h$ chip for the $k t h$ user in-phase spreading sequence

$c^{k}{ }_{q, h} \quad$ sign of the $h t h$ chip for the $k t h$ user quadrature spreading sequence

$T \quad$ chip duration

$g_{T}(t) \quad$ transmit filter impulse response

$d_{p \wedge}^{*}$ and $d_{q \mu}^{*}$ are information sequences in the case of coherent QPSK, whereas for differential QPSK they are differentially encoded version of information sequences.

The spreading sequences belong to the preferentially phased Gold codes having quasi-orthogonal cross-correlation properties in systems with a synchronization of the spreading sequences at chip level [3]. We assume that the spreading sequences in each data pulse are formed by $N$ pulses and the period of the spreading sequences is $N T_{c}$.

All frequency and timing inaccuracies at the transmitter and receiver have been neglected, since an analysis of their effects would be beyond the scope of this paper.

\section{CHANNEL MODEL}

Land-Mobile Satellite Channel

Satellite communications with land mobile terminals are plagued by severe degradation due to signal shadowing and multipath fading. Shadowing is the attenuation of the direct path, i.e. line-of-sight (LOS), over the total signal bandwidth caused by trees, buildings, hills, and mo'?tains.

Multipath fading is due to the reflected energy emanating from multiple scatterers surrounding the mobile unit. Due to their relative slowness, the environment characteristic variations may be considered constant within a small area. 
Hence, in modeling channel propagation effects, we can identify typical environment categories, build their relative models and test our communication systems separately.

A statistical propagation channel model for land mobile satellite communications, based on narrowband measurements at a single frequency, was developed by Lutz et al. [4]

Two distinct propagation link states are considered: shadowing and no shadowing.

When there is no shadowing the probability density of the instantaneous received power $P$ has the form:

$$
f_{R C E}(P)=K e^{-K(P+1)} I_{0}(2 K \sqrt{P})
$$

where $I_{0}$ is the modified Bessel function of zero order and $K$ is the ratio of LOS to average multipath power, assuming unitary LOS power.

With shadowing, the received power has the following distribution:

$$
f_{R \backslash Y Z I G H}(P)=\frac{1}{S} e^{-\frac{P}{S}}
$$

where $S$ is the average multipath power lognormally distributed. The process associated to $S$ is usually slow timevarying and therefore kept costant in our simulation.

Lutz et al. show that the model may be used only for signal bandwidths up to several tens of $\mathrm{kHz}$. This section deals with a suitable extension of the channel model given in [4] to the case of wider transmission bandwidths.

The impulse response of the channel is given by:

$$
h(t)=\sum_{k=1}^{L} \alpha_{k} e^{j \theta_{k}} \delta\left(t-\tau_{k}\right)
$$

where $\alpha, \tau, \theta$ are the gain, time delay, phase of the $k t h$ path respectively and $\delta(\theta)$ the Dirac function.

The parameters of the various paths can be found if the power delay profile [5] is known. In [6] the power delay profile is considered:

$$
P(\tau)=\frac{1}{\Delta} b e^{-\frac{\tau}{\Delta}}
$$

where $\Delta$ is the delay spread and $b$ the total multipath power. The power of path $k$ can be approximated as:

$$
b_{k}=b\left(1-e^{-\frac{T_{c}}{\Delta}}\right) e^{-\frac{(k-1) T_{c}}{\Delta}}
$$

The multipath power superimposed on the direct path is reduced with respect to the case of conventional narrowband transmission. The rest of multipath power is distributed, according to (6), among a number of resolvable paths approximately equal to:

$$
L=\left\lfloor\frac{\Delta}{T_{c}}\right\rfloor
$$

where $\lfloor x\rfloor$ is the integer part of $x$.

Simulator Architecture - We assumed a three-path model, taking into account typical values of delay spread [7] and the system transmission bandwidth.
In the unshadowed condition, the first path gain has a Rice distribution, while the others are Rayleigh distributed. In the case of a completely obstructed LOS, all three path gains are Rayleigh distributed.

Referring to the results in [4] for the statistics of the recordings made for different satellite elevations, antennas and environments, we set three typical propagation cases:

Good $(K=14 d B)$ - propagation link in an open area.

Medium $(K=5.5 \mathrm{~dB})$ - propagation link in a weakly shadowed areas or in dense scatterer areas.

$\operatorname{Bad}(K \rightarrow-\infty)$ - totally shadowed direct path

The heart of the channel model is the Rayleigh (Rice) multipath fading simulator associated to each path.

The doppler spectrum is different from zero for $|f| \leq f_{D}$; in particular we have[8]:

$$
S(f)=\frac{E^{2}}{2 \pi f_{D}}\left(1-\left(\frac{f}{f_{D}}\right)^{2}\right)^{-\frac{1}{2}}
$$

where $E$ is the root mean square (rms) value of the signal envelope and $f_{d}=V / \lambda$ is the Doppler shift corresponding to a vehicle speed $V$ and a carrier wavelength $\lambda$.

To obtain such a profile a 13th order IIR shaping filter has been used. The fading rate, or temporal variation rate of the stochastic process in the complex fading process generation, is regulated by the interaction between the filter and the interpolator according to the maximum Doppler frequency and the transmission rate of the communication system.

\section{Land Mobile Channel}

The effects observable on the received signal may be expressed in terms of superposition of two differen phenomena:

- A slow fading component. As the time variations of this fading component are relatively slow, we haven't taken them into consideration in our land mobile channel model.

- A fast fading component (multipath fading). If

wideband signaling is used, a series of delayed and attenuated echoes can be observed at channel output.

As readily observed, the situation is in every aspect similar to that of the satellite mobile channel model described in the previous section.

The model parameters used in our test are taken from [8].

\section{RECEIVER MODEL}

Referring to (1) and (4), the complex envelope of the received signal can be expressed as:

$$
S_{R}(t)=\sum_{k=1}^{L} \alpha_{k} e^{j t_{i}} S\left(t-\tau_{k}\right)+\tilde{n}(t)
$$

where $n(t)$ is a complex AWGN process with one-sided spectral noise density $N_{0}$

The assumptions we used to establish systems performance in terms of symbol error probability at the mobile unit operating upon the received signal (9), are:

- The composite signal received from the mobile unit 


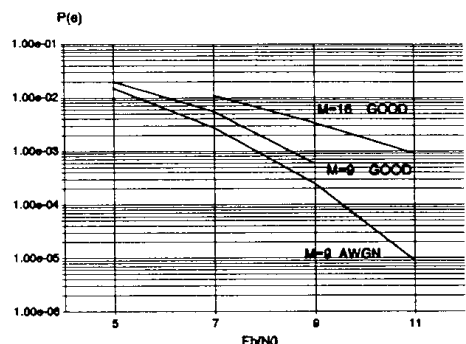

ando

Fig. 1 Probability of symbol error vs. $E_{b} / N_{o}$ for land-mobile satellite channel GOOD, coherent receiver.

comes from a single base station (satellite or terrestrial). A dedicated service channel provides the mobile unit with the spreading codes.

- A perfect chip synchronization of the incoming signal is achieved by suitable acquisition and tracking procedures.

No provision was made for channel coding or interleaving procedures, since we focused on evaluating the system intrinsic feasibility.

Coherent Receiver - The mobile unit is able to adjust its timing clock and carrier frequency to provide a perfect phase, bit, and chip synchronization with the first signal multipath component endowed with a significant power level.

The performance of the CDMA system under consideration has been derived in the case of a linear AWGN propagation channel case with real square-root raised-cosine shaping filters with a roll-off factor $\beta=0.5$. In comparing the symbol erro probability with that of the rectangular-shaped chip, no substantial differences have been highlighted. This means that even if filtering is recommended for practical applications, we may deal throughout only with the rectangular shaped chip thereby simplifying modeling and saving computation time. Noncoherent Receiver - In the case of a rapidly time-varying multipath fading channel, carrier phase recovery may be a difficult task. In order to reduce the cost of the mobile receiver, differential data demodulation may be performed.

The decision variable is now the phase of

$$
\Lambda_{s}=V_{s} V_{s-1}^{*}
$$

where $V_{s}$ is the despreader output at signaling interval $s, V_{s-1}$ is the despreader output at the signaling interval $s-1$, and ${ }^{*}$ is the complex conjugate operator.

\section{SIMULATION RESULTS AND CONSIDERATIONS}

Land-Mobile Satellite Channel

The simulation test is based on a spreading sequence set of 31 chip preferentially-phased Gold codes, working at a chiprate of $750 \mathrm{kchip} / \mathrm{s}$, which approximately corresponds to source bit rate of $50 \mathrm{kbit} / \mathrm{s}$. The channel Doppler frequency is set at $f_{D}=150 \mathrm{~Hz}$, while the power delay profile is maintained

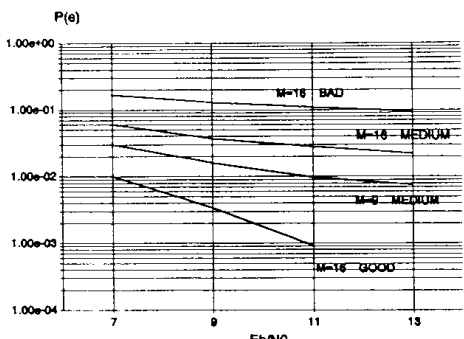

Fig. 2 Probability of symbol error vs. $E_{b} / N_{0}$ for land-mobile satellite channel MEDIUM/BAD, coherent receiver

on significant levels for approximately $4 \mu \mathrm{s}$. For the last two parameters, a better approach would have been to assign different values for different environmental conditions in the model, but owing to the lack of standard measurements, we set them to medium, i.e., plausible fixed values, preferring to differentiate the various propagation conditions only by the parameter $K$ as explained in Section II

Coherent Receiver

GOOD Channel - The symbol error probability for different operating conditions is illustrated in Fig. 1. In addition, the curve relative to a linear AWGN channel is also shown for comparison. Even with the maximum number of users $(M=16)$ simultaneously present in the channel, system performance degradation is low. Hence, we may conclude that, if the mobile terminals are localized in open areas such as highways, railways, and waterways, the considerations about a greater capacity of the CDMA scheme with respect to FDMA are fully justified.

MEDIUM Channel - The MEDIUM channel is shown in Fig. 2 , together with the BAD channel condition and a curve of the GOOD channel condition for comparison. A net performance degradation with respect to the GOOD channel and an error floor for high $E_{b} / N_{o}$ values are evident.

$B A D$ Chamel - A BAD channel results when the mobile unit is embedded in a very hostile environment where all communication systems have notable operating problems. The direct path is completely obscured and the receiver is obliged to work with the weak multipath components that reach it.

The situation is illustrated in Fig. 2 where an irreducible high error probability is shown.

Differential Receiver

$G O O D$ Channel - The system behavior is illustrated in Fig. 3. By adding $3 \mathrm{~dB}$ to the $E_{\mathrm{b}} / N_{o}$ value, it is possible to achieve the same performance as the coherent receiver.

MEDIUM Channel - As shown in Fig. 3, the full-loaded system with a heavier multipath is unable to operate correctly. Presumably, the asynchronous interference deriving from multipath delayed contributions "saturates" the more sensitive 


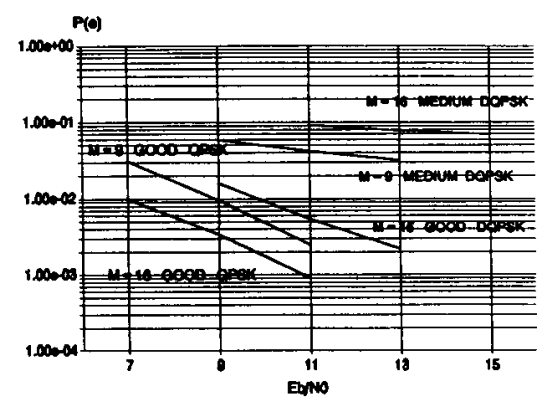

Fig. 3 Probability of symbol error vs. $E_{b} / N_{0}$ for land-mobile satellite channel GOOD, MEDIUM, differential receiver.

to noise differential demodulation system.

$B A D$ Channel - No analysis was performed for the BAD channel, since negative behavior was foreseen.

\section{Land Mobile Channel}

We chose Urban Area and Rural Area from among the propagation conditions proposed in [8] with a common maximum Doppler frequency $f_{D}=100 \mathrm{~Hz}$ and used a spreading sequence set of 127 preferentially phased Gold codes operating at a rate of $4 \mathrm{Mchip} / \mathrm{s}$.

The results are summarized in Fig. 4. Even with a low traffic flow ( $M=9$ users of 64 possible) the coherent receiver does not perform well in the Urban Area, while an acceptable symbol error probability $P(e)=10^{-2}$ is achieved in the Rural Area. A differential receiver was also tested, but performance did not prove fully satisfactory.

Hence to make terrestrial CDMA competitive with the most recent digital multiple access schemes, some diversity procedure has to be used in order to solve the problem of the propagation channel which constitutes the main impairment of system performance.

\section{RAKE RECEIVER}

We have seen earlier that, apart from the total shadowed propagation condition, CDMA behaves well for a satellite mobile radio network. However, in a terrestrial application, problems arise due to the heavier multipath presence. A practical solution to reduce the performance degradations is to use diversity techniques [5].

$R A K E$ receiver scheme - The receiver assumed in our analysis is composed of three indipendent demodulators (RAKE arms), identical to those described in Section IV. Every demodulator has one chip time-shifted despreading sequences respect to the adjacent demodulators, so that all received signal components with delays falling into a window of about three chip tin $\mathbf{s}$ can be processed. We suppose that, at the start, the first demodulator locks onto the first strong signal multipath component. The other arms sound a portion of multipath profile, searching, in a temporal fixed window, for signal

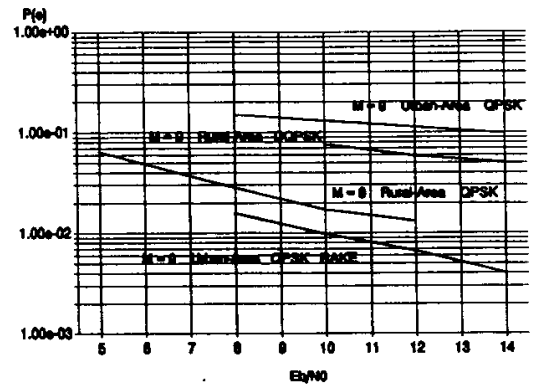

Fig. 4 Probability of symbol error vs. $E_{\downarrow} / N_{0}$ for GSM channel models.

significant contributions. The signal strength in each portion is measured by an envelope detector, whose output is integrated during a variable time interval to achieve better noise suppression. In accordance with the envelope measurements, a control unit chooses periodically the best two demodulator outputs to combine them according to an equal gain combining rule to form the decision variable.

Simulation results

Simulation results are strongly dependent on the propagation channel characterization. Considering a conventional CDMA receiver, the delayed multipath components, whether fixed or not, represent only interference while in a RAKE receiver part of them are exploited as indipendent signal contributions.

Different configurations may be considered and evaluated: a) An adaptive RAKE with indipendent arms able to follow the channel impulse response and a channel model characterized by time varying multipath components delays.

b) An adaptive RAKE with indipendent arms able to follow the channel impulse response and channel model characterized by fixed multipath components delays.

c) A fixed time referenced arms RAKE (e.g. the scheme shown previously) and a channel model characterized by time varying multipath components delays.

d) A fixed time referenced arms RAKE and a channel model characterized by fixed multipath components delays. Option a) corresponds to a "real" situation but its implementation would need accurate evaluation of feasibility of different path-tracking functions for arms time positioning.

Option b) has not the same validity as option a). Now the arms of the RAKE would periodically jump from one path to another, according to the power level measurements, instead of following a rapidly time varying propagation profile. Therefore it would have been simulated a very complex system with little correspondence to the effective operating conditions.

Option c) would offer a pessimistic outlook as the multipath components would positively contribute to the demodulation process only when synchronized with the RAKE arms. In a real situation a "continuum" of multipath components making the propagation profile is more likely to appear; therefore the fixed time delay referenced RAKE arms would probably have 


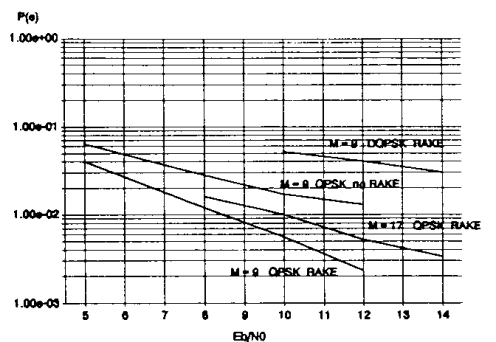

Fig. 5 Probability of symbol error vs. $E_{b} / N_{0}$ for GSM channel Rural Area, RAKE receiver.

something useful, even if weak and noisy, to demodulate all the time (see the classical approach outlined in [5, chapt. 7.5]).

Option d) has no sense if the fixed multipath time delays are different from the fixed time references of the RAKE arms, and someway predictable results if the delay coincides.

However this last statement needs a more detailed discussion.

In our simulation approach we have considered the GSM channel model suggested by [8]. In this model, the time delays associated to each multipath component are fixed. Under this assumption, the most reasonable choice was recognized to be option d) with coincident delays. At first sight the situation could simulate the case of an equivalent adaptive RAKE receiver with a perfect multipath intensity profile estimation i.e. a perfect time positioning of the RAKE arms with respect to the multipath components; this way, the scheme offers an upper bound to the performance of a very efficient adaptive RAKE receiver in a multiuser CDMA environment. A deeper investigation of the structure used in our analysis, tells us that the most important signal contributions are perfectly synchronized with the three fixed time spaced arms of the RAKE. This case simulates the situation where a multipath intensity profile, or equivalently a continuum of multipath components, is sounded with a resolution of $T_{c}$, where a $T_{c}$ is the duration of a chip. Therefore, our analysis can be considered as an extension of the results given in [5] to consider the case of a CDMA multiple access environment with well-established and standardized channel model parameters.

Coherent receiver - We suppose that in the two demodulators chosen by the control unit a perfect carrier recovery is performed. The results for the Urban Area are illustrated in Fig. 4; the performance improvement is evident.

In the case of Rural Area, Fig. 5, the improvement is not so relevant, but still appreciable and an increase in capacity is possible at the expence of a little increase in system architecture complexity.

Differential receiver - Every chosen arm performs a differential demodulation before implementing the unity weighted combining. In the Urban Area the system has a very bad behavior, probably due to the presence, in every chosen arm, of strong aysnchronous interference that saturates the differential demodulation system. In Rural Area, as illustrated in Fig. 5, the RAKE improvement in performance is almost negligible with respect to the results shown in Fig. 4. The last one is a quite interesting result; if we have to deal with a mobile communication link characterized by a Rice LOS propagation path, even in presence of strong delayed multipath components, a differential demodulation scheme and a CDMA multiple access, the real effectiveness of a simple RAKE receiver appears questionable.

\section{CONCLUSIONS}

In this paper the modeling and performance evaluation of different CDMA schemes suitable for application in both terrestrial and satellite mobile networks have been considered.

Extensive computer simulation runs of perfectly coherent QPSK-CDMA and DQPSK-CDMA have showed that if the mobile terminal is localized in open areas, system performance degradation caused by multipath interference is not too large even for very high load conditions. In particular DQPSKCDMA seems a good choice for a simple, reliable and efficient with regard to multipath, multiple access scheme.

In more difficult, but not totally shadowed, propagation conditions, QPSK-CDMA is still good, whereas DQPSKCDMA can operate correctly only for a low traffic.

As far as land mobile communications are concerned, system performance is not so good as in the low shadowed satellite communications. The issues concerning a realistic and reliable computer simulation of a RAKE receiver have been discussed.

More realistic channel models and more realistic and sophisticated CDMA receivers, conventional or RAKE, are currently under study.

REFERENCES

[1] K. S. Gilhousen, I. M. Jacobs, R. Padovani, L. A. Weaver Jr., "Increased Capacity Using CDMA for Mobile Satellite Communication", IEEE Joumal on selected Areas in Communications, VOL. 8, No. 4 May 1990.

[2] G. L. Turin, The Effects of Multipath and Fading on the Performance of Direct-Sequence CDMA Systems", IEEE Journal on Selected Areas in Communications, VOL. SAC-2, No.4, July 1984

[3] R. De Gaudenzi, C. Elia, R. Viola, "Band-limited Quasi-synchronous CDMA: A novel Satellite Access Technique for Mobile and Personal Communication Systems", Journal on Selected Areas in Communications, Communication Systems", Journ
VOL. 10, No. 2, Februay 1992.

[4] E. Lutz, D. Cygan, M. Dippold, F. Dolainsky, W. Papke, " The Land Mobile Satellite Communication Channel - Recordings, Statistics and Channel Model ${ }^{n}$, IEEE Transactions on Vehicular Technology, VOL 40, No. 2, May 1991.

[5] J. G.Proakis, "Digital Communications", Second Edition, McGRAWHILL, 1989

[6] R. Van Nee, H. S. Misser, R. Prasad, "Direct-Sequence Spread-Spectrum in a Shadowed Rician Fading Land Mobile Satellite Channel", IEEE Joumal on Selected Areas in Communications, VOL. 10, No.2, February 1992.

[7] R. H. Raekken, H. Langas, G. Lovnes, S. E. Paulsen, Wideband Impulse Response Messurements at $900 \mathrm{MHz}$ ", Globeco 1991.

[8] GSM Recommendation 05.05, Transmission and Reception. 